\title{
Computational-Fluid-Dynamics Solver with Preconditioned Method for Aerodynamic Simulation of High-Lift Configuration
}

\author{
Zhongyu Liu, ${ }^{*}$ Wen Liu,, \pm Xueyuan Nie, \pm and Guowei Yang $\underline{\S}$ \\ Chinese Academy of Sciences, 100190 Beijing, People's Republic of China
}

DOI: $10.2514 / 1 . C 033612$

\begin{abstract}
A preconditioned method with characteristic boundary conditions for Navier-Stokes equations is implemented for flowfield analysis of a high-lift configuration at low Mach number. A preconditioned Harten-Lax-van Leer-EinfeldtWada scheme is adopted for the spatial discretization, and the dissipation term of which is rededuced to reduce the overlarge numerical dissipation. Preconditioned characteristic boundary conditions for the far field are derived based on the Weiss-Smith preconditioner and are demonstrated to be more reliable and accurate when coupled with the preconditioned method. A preconditioned Lower-Upper Symmetric Gauss-Seidel implicit time-marching method is modified according to the preconditioned Jacobian matrix. The applications of the current method on both two-and three-dimensional high-lift configurations indicate that the use of the preconditioned spatial and time discrete schemes and the derived preconditioned characteristic boundary conditions is capable of improving the robustness, efficiency, and accuracy of the computational-fluid-dynamics solver for low-Mach-number aerodynamic simulations.
\end{abstract}

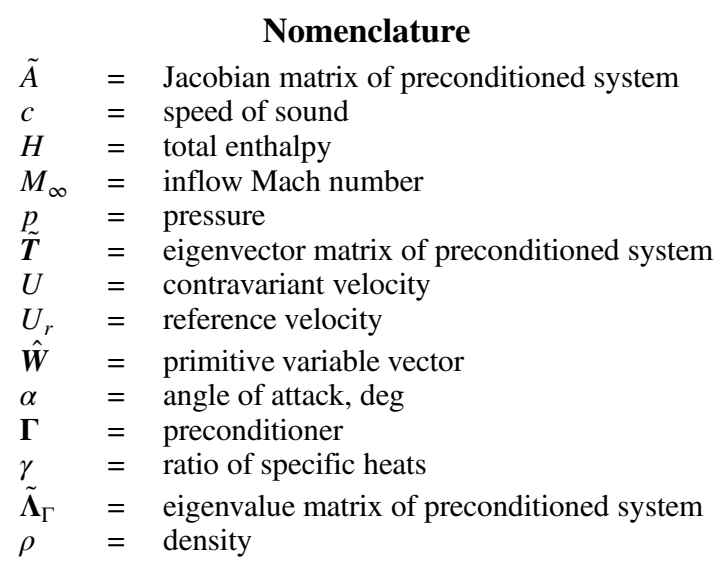

Subscripts

$b \quad=\quad$ value on boundary

$d=$ value in computational domain

$\boldsymbol{\Gamma}=$ value in preconditioned system

$\infty=$ freestream value

\section{Introduction}

$\mathbf{T}$ HE efficiency of high-lift devices is critical for the performance of civil aircraft, especially for the payload limits. The multielement configuration complicates the flow physics around the high-lift devices with massive flow separations, boundary-layer transitions, and wake interactions, which makes accurate aerodynamic

Received 30 June 2015; revision received 8 September 2015; accepted for publication 1 October 2015; published online 23 December 2015. Copyright (C) 2015 by the American Institute of Aeronautics and Astronautics, Inc. All rights reserved. Copies of this paper may be made for personal and internal use, on condition that the copier pay the per-copy fee to the Copyright Clearance Center (CCC). All requests for copying and permission to reprint should be submitted to CCC at www.copyright.com; employ the ISSN 00218669 (print) or 1533-3868 (online) to initiate your request.

*Research Assistant, Key Laboratory for Mechanics in Fluid Solid Coupling Systems, Institute of Mechanics; liuzhongyu @imech.ac.cn.

${ }^{\dagger}$ Research Associate, Key Laboratory for Mechanics in Fluid Solid Coupling Systems, Institute of Mechanics; liuwen@imech.ac.cn.

*Research Associate, Key Laboratory for Mechanics in Fluid Solid Coupling Systems, Institute of Mechanics; niexueyuan@imech.ac.cn.

${ }^{\S}$ Research Professor, Key Laboratory for Mechanics in Fluid Solid Coupling Systems, Institute of Mechanics; gwyang@imech.ac.cn. predictions using the computational fluid dynamics (CFD) solver quite challenging.

CFD solvers based on Navier-Stokes (NS) equations in the conservative form have been successfully applied in the simulations of transonic and supersonic flows. However, the use of those solvers for low-Mach-number flows often has convergence problems due to the large disparity between convective and acoustic wave velocities and therefore does not work well for incompressible flow problems. Inspired by Chorin's [1] artificial compressibility method, Turkel [2] proposed a preconditioned method for the lowMach-number flow problem. The basic idea of the preconditioned method is to reduce the disparity between the convective and acoustic wave velocities through the modification of the eigensystem of the governing equations, which can be achieved by multiplying the preconditioning matrix to the time-derivative term. Various forms of the preconditioning matrices are proposed by Turkel [2], van Leer et al. [3] , Weiss and Smith [4]], and Venkatakrishnan [్]] for inviscid and viscous flows.

Unfortunately, the improvements in the convergence rate and accuracy are obtained by reducing the robustness of the solver, which becomes an inhibitory factor of the preconditioned methods for engineering applications. The stability of the preconditioned method is sensitive to the preconditioned parameter, especially in stagnationpoint regions and boundary-layer regions. Choi and Merkle [6] suggested reducing preconditioning in such regions to gain better robustness. Many efforts have been focused on defining limiting factors and prescribing cutoff values for the preconditioned parameter to increase the stability of preconditioned methods [ $6-10]$ ].

The boundary conditions (BCs) on the far fields are another important factor that may significantly affect the stability and accuracy of the preconditioned method. Investigations [11-13] show that the performance of the flow solver can be deteriorated by the reflection of spurious waves on the truncated domain boundaries; therefore, the implementation of nonreflecting boundary conditions for the farfield boundaries is necessary. One typical solution is the use of Riemann BCs, which are based on the one-dimensional characteristics to eliminate the spurious reflecting waves. A characteristic form of the preconditioned system of equations has been given $[\underline{11}, 14,15]$, and preconditioned characteristic BCs are proposed for the preconditioned methods proposed by Ref. [12]. Simplified BCs $[\underline{11}, \underline{16}]$ and standard Riemann BCs [12] are adopted on the far-field boundaries in many implementations of the preconditioned method. However, the simplified BCs and the standard Riemann BCs may not yield the optimal performance for the preconditioned system because the characteristic features of the governing equations have been altered by the preconditioning. It is essential then that the preconditioned characteristic BCs are used for the specific preconditioner to ensure the stability and accuracy of the solver. 
In the current study, we developed a cell-centered, finite-volume, three-dimensional Navier-Stokes flow solver with the Weiss-Smith preconditioner. A preconditioned Harten-Lax-van Leer-EinfeldtWada (HLLEW) scheme is adopted for the spatial discretization $[17,18]$, and the dissipation term of which is rederived to reduce the overlarge numerical dissipation. Preconditioned characteristic BCs based on the Weiss-Smith preconditioner are derived at the far-field boundaries, the effect of which is assessed by the comparisons with the simplified BCs and the standard Riemann BCs. The preconditioned Lower-Upper Symmetric Gauss-Seidel (LU-SGS) implicit time-marching method is adopted to improve the convergence rate of the solution. To evaluate the stability and accuracy of the solver, flows over the two-dimensional National Aerospace Laboratory (NLR) 7301 airfoil with flap and threedimensional high-lift trap-wing configuration [19-21] in the First AIAA CFD High-Lift Prediction Workshop [22] are analyzed and validated by the experimental data.

\section{Preconditioned Formulation}

The preconditioned method for Reynolds-averaged NavierStokes (RANS) equations can be obtained by multiplying the preconditioner $\boldsymbol{\Gamma}$ to the time derivative terms in NS equations. It can be shown that, although the propagation velocities of the equation system will be changed, the steady solutions remain unaffected. The preconditioned governing equations can be written as follows:

$$
\boldsymbol{\Gamma} \frac{\partial \hat{\boldsymbol{W}}}{\partial t}+\frac{\partial \hat{\boldsymbol{E}}}{\partial \xi}+\frac{\partial \hat{\boldsymbol{F}}}{\partial \eta}+\frac{\partial \hat{\boldsymbol{G}}}{\partial \zeta}=\frac{\partial \hat{\boldsymbol{E}}_{v}}{\partial \xi}+\frac{\partial \hat{\boldsymbol{F}}_{v}}{\partial \eta}+\frac{\partial \hat{\boldsymbol{G}}_{v}}{\partial \zeta}
$$

where $\hat{W}$ is the primitive variable vector and has various forms depending on different choices of the preconditioner $\boldsymbol{\Gamma}$. In this paper, the preconditioner proposed by Weiss and Smith [4] is adopted:

$$
\boldsymbol{\Gamma}=\left(\begin{array}{ccccc}
\Theta & 0 & 0 & 0 & \rho_{T} \\
\Theta u & \rho & 0 & 0 & \rho_{T} u \\
\Theta v & 0 & \rho & 0 & \rho_{T} v \\
\Theta w & 0 & 0 & \rho & \rho_{T} w \\
\Theta H-1 & \rho u & \rho v & \rho w & \rho_{T} H+\rho C_{p}
\end{array}\right)
$$

Accordingly, the primitive variable vector is taken as $\hat{\boldsymbol{W}}=$ $\left(\begin{array}{lllll}p & u & v & w & T\end{array}\right)^{T}$. In the previous preconditioner,

$$
\begin{gathered}
\rho_{T}=\left.\frac{\partial \rho}{\partial T}\right|_{p} \\
\Theta=\left(\frac{1}{U_{r}^{2}}-\frac{\rho_{T}}{\rho C_{p}}\right)
\end{gathered}
$$

and $U_{r}$ is the reference velocity given by

$$
U_{r}=M_{r} c
$$

where $M_{r}$ is the preconditioner parameter and $c$ is the speed of sound.

The stability of the preconditioned method is sensitive to the preconditioner parameter $M_{r}$, especially in the stagnation-point regions and the boundary-layer regions. Many efforts have been focused on defining limiting factors and prescribing cutoff values for $M_{r}$ to increase the stability of the preconditioned method [7-10]. However, for a cell-centered finite-volume solver, the $M_{r}$ on the interface of adjacent cells is interpolated based on the centroids of neighbor cells. The $M_{r}$ sometimes has different values on each side of the interface, especially in the regions where the cutoff and limiting works. This disparity of $M_{r}$ values across the interface will significantly reduce the robustness of the flow solver. The uniform preconditioner parameter is used to eliminate the inconsistency caused by discretization and to enhance the stability of the solver.

\section{Numerical Discretization}

\section{A. Preconditioned Spatial Discretization Scheme}

The preconditioned governing equations of Eq. (1) are discretized in space using a cell-centered finite-volume method based on the structured meshes. The discrete inviscid flux vectors on cell faces are evaluated by the preconditioned HLLEW flux-difference splitting scheme. In the $\xi$ direction, for example, the flux vector with the preconditioned HLLEW scheme in RANS equations is given by

$$
\hat{\boldsymbol{E}}_{i+1 / 2, j, k}=\frac{1}{2}\left(\hat{\boldsymbol{E}}_{i, j, k}+\hat{\boldsymbol{E}}_{i+1, j, k}\right)-\frac{1}{2}|\tilde{\boldsymbol{A}}|_{i+1 / 2, j, k}\left(\hat{\boldsymbol{Q}}_{i+1, j, k}-\hat{\boldsymbol{Q}}_{i, j, k}\right)
$$

where the subscripts $L$ and $R$ represent the left and right sides of the cell face, respectively. The Jacobian matrix $\tilde{\boldsymbol{A}}$ is given by $\partial \hat{\boldsymbol{E}} / \partial \hat{\boldsymbol{Q}}$. In the preconditioned governing equations, eigenvalues of the equation system are adjusted, and thus the dissipation term in Eq. (9) needs to be modified accordingly:

$$
|\tilde{\boldsymbol{A}}| \Delta \hat{\boldsymbol{Q}}=\boldsymbol{\Gamma}\left(\tilde{\boldsymbol{T}}\left|\tilde{\boldsymbol{\Lambda}}_{\Gamma}\right| \tilde{\boldsymbol{T}}^{-1} \hat{\boldsymbol{W}}\right)
$$

where $\tilde{\boldsymbol{\Lambda}}_{\Gamma}$ is the diagonal matrix of the preconditioned system, and $\tilde{\boldsymbol{T}}$ is the eigenvectors matrix. The eigenvalues are modified to reduce the numerical dissipative of the scheme and to enhance the resolution at contact discontinuities [17]:

$$
\overline{\boldsymbol{\Lambda}}_{\Gamma}=\frac{b_{R}^{+}+b_{L}^{-}}{b_{R}^{+}-b_{L}^{-}} \boldsymbol{\Lambda}-2 \frac{b_{R}^{+} b_{L}^{-}}{b_{R}^{+}-b_{L}^{-}} \boldsymbol{I}
$$

$$
\begin{gathered}
\tilde{\Lambda}_{\Gamma}=\operatorname{diag}\left[\bar{\lambda}_{1}-2 \delta \min \left(b_{R}^{+}, b_{L}^{-}\right), \quad \bar{\lambda}_{2}-2 \delta \min \left(b_{R}^{+}, b_{L}^{-}\right),\right. \\
\left.\bar{\lambda}_{3}-2 \delta \min \left(b_{R}^{+}, b_{L}^{-}\right), \bar{\lambda}_{4}, \bar{\lambda}_{5}\right]
\end{gathered}
$$

where

$$
\delta=\min \left(\frac{\tilde{\rho}}{\left|\sigma_{1}\right|}, \frac{1}{2}\right)
$$

$$
\sigma_{1}=\Delta \rho-\frac{\Delta p}{\tilde{c}^{2}}
$$

and $b_{R}^{+}$and $b_{L}^{-}$stand for wave propagating speeds:

$$
\begin{aligned}
& b_{R}^{+}=\max \left(U^{\prime}+C^{\prime}, U_{R}^{\prime}+C_{R}^{\prime}, 0\right) \\
& b_{L}^{-}=\min \left(U^{\prime}-C^{\prime}, U_{L}^{\prime}-C_{L}^{\prime}, 0\right)
\end{aligned}
$$

For the isentropic flow, the scheme will be reduced into the standard upwind-biased Roe scheme. In nonlinear regions, the scheme satisfies the entropy and positive conditions and can enhance the resolution to a level comparing with the Roe scheme.

\section{B. Time-Marching Method}

For steady-state problems, the time derivative term is discretized by the first-order forward-difference scheme. Inviscid flux terms are discretized implicitly, and the explicit treatment is adopted for the viscous flux terms. Thus, a semidiscrete governing equation for the preconditioned system is obtained:

$\boldsymbol{\Gamma}_{I, J, K} \frac{\hat{\boldsymbol{W}}_{i, j, k}^{n+1}-\hat{\boldsymbol{W}}_{i, j, k}^{n}}{\Delta \tau}+\delta(\hat{\boldsymbol{E}}+\hat{\boldsymbol{F}}+\hat{\boldsymbol{G}})_{i, j, k}^{n+1}=\delta\left(\hat{\boldsymbol{E}}_{v}+\hat{\boldsymbol{F}}_{v}+\hat{\boldsymbol{G}}_{v}\right)_{i, j, k}^{n}$

Then, we can linearize the inviscid flux vectors in Eq. (9) and neglect high-order terms; the resultant equations can be written as follows: 


$$
\begin{aligned}
& \frac{\Gamma_{i, j, k}^{n}}{\Delta t} \Delta \hat{\boldsymbol{W}}_{i, j, k}^{n}+\left(\boldsymbol{A}_{\Gamma} \Delta \hat{\boldsymbol{W}}\right)_{i+1 / 2, j, k}^{n} \\
& \quad-\left(\boldsymbol{A}_{\Gamma} \Delta \hat{\boldsymbol{W}}\right)_{i-1 / 2, j, k}^{n}+\left(\boldsymbol{B}_{\Gamma} \Delta \hat{\boldsymbol{W}}\right)_{i, j+1 / 2, k}^{n} \\
& \quad-\left(\boldsymbol{B}_{\Gamma} \Delta \hat{\boldsymbol{W}}\right)_{i, j-1 / 2, k}^{n}+\left(\boldsymbol{C}_{\Gamma} \Delta \hat{\boldsymbol{W}}\right)_{i, j, k+1 / 2}^{n}-\left(\boldsymbol{C}_{\Gamma} \Delta \hat{\boldsymbol{W}}\right)_{i, j, k-1 / 2}^{n}=\hat{\boldsymbol{R}}_{i, j, k}^{n}
\end{aligned}
$$

In the previous equation, $\hat{\boldsymbol{R}}_{i, j, k}^{n}=\delta(\hat{\boldsymbol{E}}+\hat{\boldsymbol{F}}+\hat{\boldsymbol{G}})_{i, j, k}^{n}-\delta\left(\hat{\boldsymbol{E}}_{v}+\right.$ $\left.\hat{\boldsymbol{F}}_{v}+\hat{\boldsymbol{G}}_{v}\right)_{i, j, k}^{n}$ is the residual vector. $\boldsymbol{A}_{\Gamma}, \boldsymbol{B}_{\Gamma}$, and $\boldsymbol{C}_{\Gamma}$ are the Jacobian matrices for the inviscid flux vectors $\hat{\boldsymbol{E}}, \hat{\boldsymbol{F}}$, and $\hat{\boldsymbol{G}}$, based on the primitive variables $\hat{\boldsymbol{W}}$. Decomposing the left-hand side in Eq. (17) with an approximate lower upper decomposition method, using the symmetric Gauss-Seidel method, the linearized equation can be solved iteratively in a two-step process. For viscous flow, the contribution of the viscous flux terms is taken into consideration to ensure the stability of the method.

\section{Far-Field Boundary Conditions}

Numerical simulation of external flows is usually conducted within a bounded domain. Because of its elliptic nature, the preconditioned system for subsonic flow is sensitive to the far-field BCs. Simplified $\mathrm{BCs}$ and standard Riemann BCs are adopted on the far-field boundaries in many implements of the preconditioned method. However, these two types of BCs may not yield the optimal performance for the preconditioned system because the characteristic features of the governing equations have been altered by the preconditioning. Therefore, implementation of nonreflecting BCs for the far-field boundaries is necessary. Preconditioned characteristic BCs based on the WeissSmith preconditioner are derived at the far-field boundaries, the effect of which is assessed by the comparisons with the simplified BCs and the standard Riemann BCs.

Referring to the preconditioned governing equations in Eq. (1), the one-dimensional governing equation on the boundary can be written as follows:

$$
\frac{\partial \boldsymbol{W}}{\partial t}+\boldsymbol{\Gamma}^{-1} \boldsymbol{A}_{\Gamma} \frac{\partial \boldsymbol{W}}{\partial n}=0
$$

where $n$ is normal distant to the boundary. Considering that $\boldsymbol{\Gamma}^{-1} \boldsymbol{A}_{\Gamma}=\boldsymbol{R}_{\Gamma} \boldsymbol{\Lambda}_{\Gamma} \boldsymbol{R}_{\Gamma}^{-1}$, Eq. (11) can be transformed as

$$
\boldsymbol{R}_{\Gamma}^{-1} \frac{\partial \boldsymbol{W}}{\partial t}+\boldsymbol{\Lambda}_{\Gamma} \boldsymbol{R}_{\Gamma}^{-1} \frac{\partial \boldsymbol{W}}{\partial n}=0
$$

For the Weiss-Smith preconditioner, the eigenvector matrix is determined as

$$
\boldsymbol{R}_{\Gamma}^{-1}=\left(\begin{array}{ccccc}
-\frac{1}{\rho \boldsymbol{C}_{\mathrm{p}}} & 0 & 0 & 0 & 1 \\
0 & -\frac{n_{x} n_{z}}{n^{2}} & -\frac{n_{y} n_{z}}{n^{2}} & \frac{n_{x}^{2}+n_{y}^{2}}{n^{2}} & 0 \\
0 & -\frac{n_{x} n_{y}}{n^{2}} & \frac{n_{x}^{2}+n_{z}^{2}}{n^{2}} & -\frac{n_{y} n_{z}}{n^{2}} & 0 \\
\frac{r}{4 \rho c^{\prime} \boldsymbol{C}_{\mathrm{p}}} & -\frac{n_{x} U_{r}^{2}}{2 c^{\prime} \boldsymbol{C}_{\mathrm{p}}} & -\frac{n_{y} U_{r}^{2}}{2 c^{\prime} \boldsymbol{C}_{\mathrm{p}}} & -\frac{n_{z} U_{r}^{2}}{2 c^{\prime} \boldsymbol{C}_{\mathrm{p}}} & 0 \\
-\frac{s}{4 \rho c^{\prime} \boldsymbol{C}_{\mathrm{p}} Q} & \frac{n_{x} U_{r}^{2}}{2 c^{\prime} \boldsymbol{C}_{\mathrm{p}}} & \frac{n_{y} U_{r}^{2}}{2 c^{\prime} \boldsymbol{C}_{\mathrm{p}}} & \frac{n_{z} U_{r}^{2}}{2 c^{\prime} \boldsymbol{C}_{\mathrm{p}}} & 0
\end{array}\right)
$$

Substituting $\boldsymbol{R}_{\Gamma}^{-1}$ into Eq. (12), the preconditioned compatibility equations and the corresponding characteristic line equations are obtained:

$$
\begin{aligned}
\mathrm{d} R_{1} & =\frac{\mathrm{d} p}{\rho C_{p}}+\mathrm{d} T, \quad \frac{\mathrm{d} x_{1}}{\mathrm{~d} t}=U \\
\mathrm{~d} R_{2} & =n_{x} n_{z} \mathrm{~d} u+n_{y} n_{z} \mathrm{~d} v-\left(n_{x}^{2}+n_{y}^{2}\right) \mathrm{d} w, \quad \frac{\mathrm{d} x_{2}}{\mathrm{~d} t}=U \\
\mathrm{~d} R_{3} & =n_{x} n_{y} \mathrm{~d} u-\left(n_{x}^{2}+n_{z}^{2}\right) \mathrm{d} v+n_{y} n_{z} \mathrm{~d} w, \quad \frac{\mathrm{d} x_{3}}{\mathrm{~d} t}=U \\
\mathrm{~d} R_{4} & =\frac{2 c^{\prime}-\left(M_{r}^{2}-1\right) U}{2 \rho M_{r}^{2} c^{2}} \mathrm{~d} p-\mathrm{d} U, \quad \frac{\mathrm{d} x_{4}}{\mathrm{~d} t}=U^{\prime}-c^{\prime} \\
\mathrm{d} R_{5} & =\frac{2 c^{\prime}+\left(M_{r}^{2}-1\right) U}{2 \rho M_{r}^{2} c^{2}} \mathrm{~d} p+\mathrm{d} U, \quad \frac{\mathrm{d} x_{5}}{\mathrm{~d} t}=U^{\prime}+c^{\prime}
\end{aligned}
$$

where $R_{1}, R_{2}, R_{3}, R_{4}, R_{5}$ are the characteristic invariants of the preconditioned governing equations. For calorically perfect gas, the first compatibility equation can be integrated as

$$
\frac{p}{\rho^{\gamma}}=C_{0}
$$

It shows that entropy remains conserved along the first characteristic line. From Eq. (15), we have the following:

$$
\mathrm{d} p=\frac{2 \gamma}{\gamma-1} \frac{p}{c} \mathrm{~d} c
$$

Then, the last two compatibility equations can be rearranged as follows:

$$
\begin{aligned}
\mathrm{d} R_{4} & =\frac{2 c^{\prime}-\left(M_{r}^{2}-1\right) U}{M_{r}^{2} c(\gamma-1)} \mathrm{d} c-\mathrm{d} U \\
\mathrm{~d} R_{5} & =\frac{2 c^{\prime}+\left(M_{r}^{2}-1\right) U}{M_{r}^{2} c(\gamma-1)} \mathrm{d} c+\mathrm{d} U
\end{aligned}
$$

For subsonic flow, the propagation velocity on the characteristic line 4 is less than zero; thus, the flow variables on boundary are determined by the freestream conditions. As to characteristic line 5, the flow variables depend on those in the computational domain because propagation velocity is positive. Hence, the variables on the boundary can be determined as

$$
\begin{aligned}
& U_{b}-U_{\infty}-\frac{2 c_{\infty}^{\prime}-\left(M_{r}^{2}-1\right) U_{\infty}}{M_{r}^{2} c_{\infty}(\gamma-1)}\left(c_{b}-c_{\infty}\right)=0 \\
& U_{b}-U_{d}-\frac{2 c_{i}^{\prime}-\left(M_{r}^{2}-1\right) U_{i}}{M_{r}^{2} c_{i}(\gamma-1)}\left(c_{b}-c_{d}\right)=0
\end{aligned}
$$

Solving the previous linear equations, the contravariant velocity and the speed of sound on the boundary can be obtained:

$$
\begin{aligned}
U_{b} & =\frac{A B\left(c_{d}-c_{\infty}\right)+B U_{\infty}+A U_{d}}{A+B} \\
c_{b} & =\frac{A c_{\infty}+B c_{d}-U_{\infty}+U_{d}}{A+B}
\end{aligned}
$$

with

$$
\begin{aligned}
A & =\frac{2 c_{\infty}^{\prime}-\left(M_{r}^{2}-1\right) U_{\infty}}{M_{r}^{2} c_{\infty}(\gamma-1)} \\
B & =\frac{2 c_{d}^{\prime}-\left(M_{r}^{2}-1\right) U_{d}}{M_{r}^{2} c_{d}(\gamma-1)}
\end{aligned}
$$

For three-dimensional problems, the definition of each velocity component is required. According to the compatibility equations of the characteristic lines 2 and 3, combining the definition of the contravariant velocity: 


$$
\begin{aligned}
& n_{x} n_{z} \mathrm{~d} u+n_{y} n_{z} \mathrm{~d} v-\left(n_{x}^{2}+n_{y}^{2}\right) \mathrm{d} w=0 \\
& n_{x} n_{y} \mathrm{~d} u-\left(n_{x}^{2}+n_{z}^{2}\right) \mathrm{d} v+n_{y} n_{z} \mathrm{~d} w=0 \\
& n_{x} \mathrm{~d} u+n_{y} \mathrm{~d} v+n_{z} \mathrm{~d} w=\mathrm{d} U
\end{aligned}
$$

Solving the previous linear equations, the following relation is obtained:

$$
\begin{aligned}
\mathrm{d} u & =n_{x} \mathrm{~d} U \\
\mathrm{~d} v & =n_{y} \mathrm{~d} U \\
\mathrm{~d} w & =n_{z} \mathrm{~d} U
\end{aligned}
$$

As a result, the velocity components on the boundary can be determined as

$$
\begin{aligned}
u_{b} & =u_{\mathrm{ref}}+n_{x}\left(U_{b}-U_{\mathrm{ref}}\right) \\
v_{b} & =v_{\mathrm{ref}}+n_{y}\left(U_{b}-U_{\mathrm{ref}}\right) \\
w_{b} & =w_{\mathrm{ref}}+n_{z}\left(U_{b}-U_{\mathrm{ref}}\right)
\end{aligned}
$$

By setting the preconditioned parameter $M_{r}=1$, the expression of flow variables for the preconditioned system recovers to that of the standard Riemann BCs.

\section{Results and Discussion}

In this section, three different simulations are presented to evaluate the accuracy, robustness, and efficiency of the current preconditioned method. First, the Royal Aircraft Establishment (RAE) 2822 transonic flow was tested to assess the effect of the derived preconditioned characteristic $\mathrm{BCs}$ by the comparisons with the simplified BCs and the standard Riemann BCs. Then, the simulations of the twodimensional (2-D) NLR 7301 airfoil with flap and the threedimensional (3-D) high-lift trap-wing configuration are presented as applications of our method for 2-D and 3-D geometries.

In the simulations, the two-equation $k-\omega$ shear-stress transport turbulence model is adopted to compute the eddy viscosity coefficient. In each iteration, the turbulence model equations are solved decoupled from the governing equations. Both groups of the equations are solved with the same time step to ensure that they are advanced synchronously. The convergence rate is measured by the L2 norm of the density residual, which is determined by Eq. (15).

\section{A. Transonic Flow over Royal Aircraft Establishment 2822 Airfoil}

The computational domain of the RAE 2822 airfoil case is shown in Fig. 1. A C-type structured mesh is used, and the mesh size is $64 \times 36 \overline{8}$. The freestream Mach number is $M_{\infty}=0.729$, Reynolds

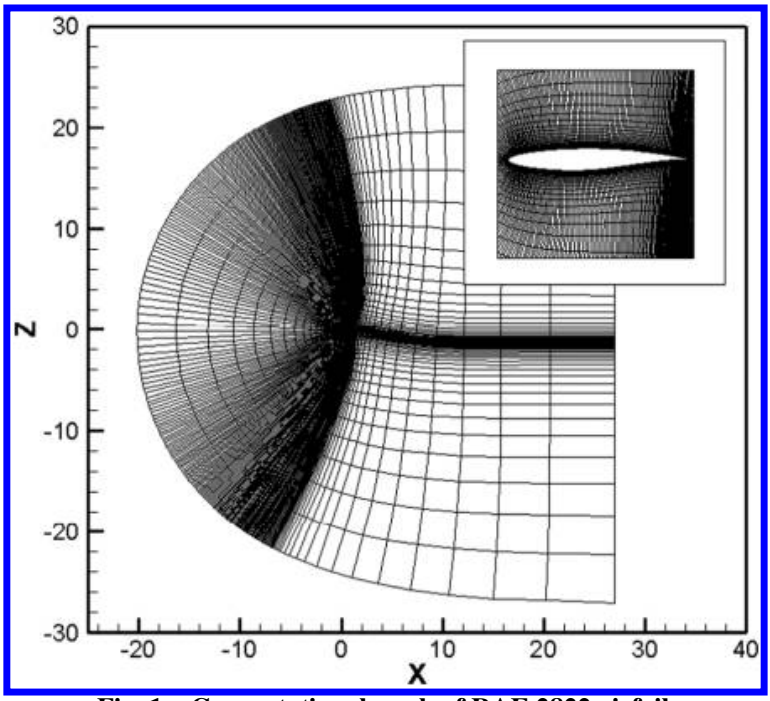

Fig. 1 Computational mesh of RAE 2822 airfoil. number based on the chord length is $6.5 \times 10^{6}$, and the angle of attack (AOA) is $2.31 \mathrm{deg}$. The minimum distance from the far-field boundary to the airfoil is more than 20 times the chord length.

The convergence behaviors of different boundary conditions are displayed in Fig. 2. The residuals in this figure are computed with the preconditioner parameter of $M_{r}=0.5$. It is shown that the preconditioned method with the simplified BCs has the slowest convergence rate because the reflection waves from the far-field boundaries affect the flowfield near the wall. The waves from the far field and the wall reflect several times until the flowfield converges, which may significantly slow down the convergence rate of the solution. The use of the preconditioned characteristic BCs can speed up the convergence rate significantly, though efficiency improvement is slightly less than that of the standard Riemann BCs for the transonic flows. The preconditioned method reduces the eigenvalues of the governing equations; thus, the spreading speed of the wall disturbance decreases accordingly, as a result of which the convergence rate of preconditioned characteristic BCs is not as efficient as that of standard Riemann BCs.

The pressure distributions on the wall surface are validated by the experimental data in Fig. 3. On the lower surface, the pressure predictions are in good agreement with the measurement data for all the three BCs. On the upper surface, disparities appear near the leading edge and in the nonlinear region. At the leading edge, the preconditioned characteristic BCs and the standard Riemann BCs

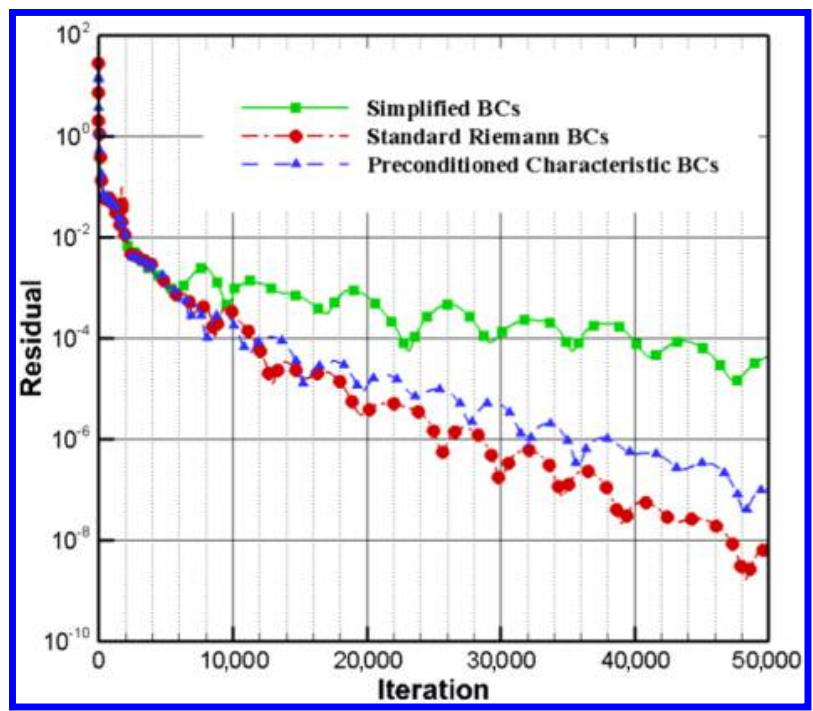

Fig. 2 Comparison of convergence rate with different boundary conditions.

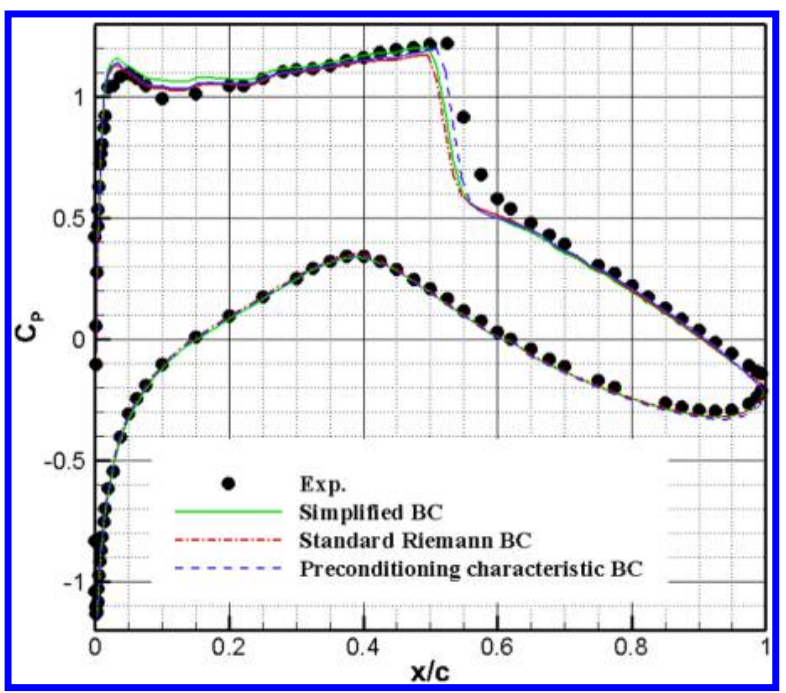

Fig. 3 Comparison of pressure coefficient distribution on surface. 


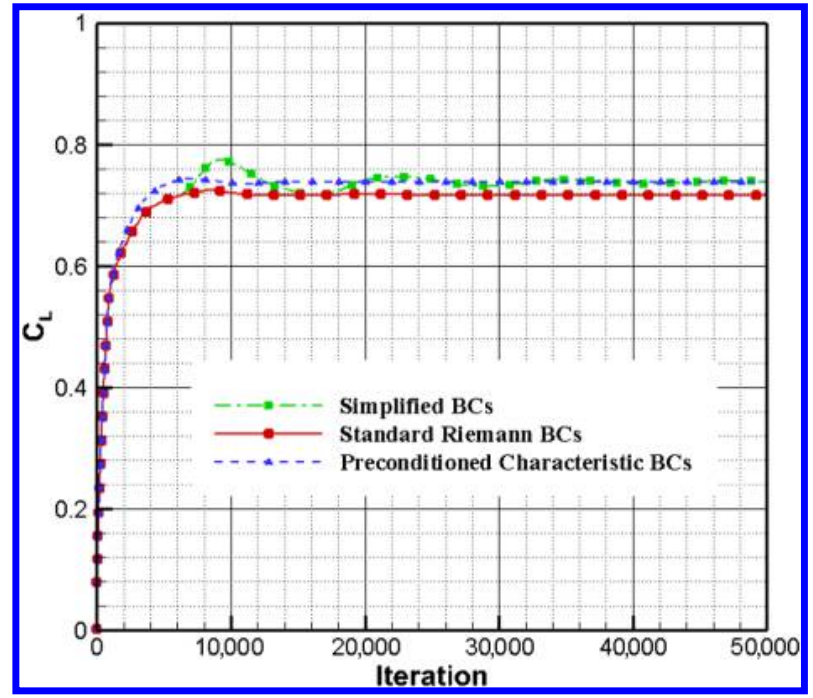

Fig. 4 Convergence history of lift coefficient with different boundary conditions.

provide better pressure predictions than the simplified BCs. In the shock region, the preconditioned characteristic BCs have the best performance in capturing the shock wave compared to the experimental data.

Figure 4 depicts the convergence history of lift coefficients calculated by simulations with different BCs for RAE 2822 transonic flow. The predicted lift coefficient using the preconditioned characteristic BCs and the simplified BCs are 0.7374 and 0.7383 , respectively. The relative error of these two simulation results with respect to the experimental data, which is 0.7429 , is less than $1 \%$. However, the lift coefficient predicted using the standard Riemann BCs is 0.7170 , which is not as accurate as that of the preconditioned characteristic BCs, because the standard Riemann BCs do not eliminate the boundary reflection of the spurious waves for the preconditioned system.

The study shows that the BCs on the far-field boundaries have an important influence on the performance of the flow solver. The preconditioned characteristic BCs eliminate the reflection spurious waves on the far-field boundaries; thus, the solution efficiency is improved, and more accurate results are obtained.

\section{B. National Aerospace Laboratory 7301 Wing with Flap}

Simulation results for low-speed flow over a two-dimensional multi-element high-lift configuration are presented in this section.

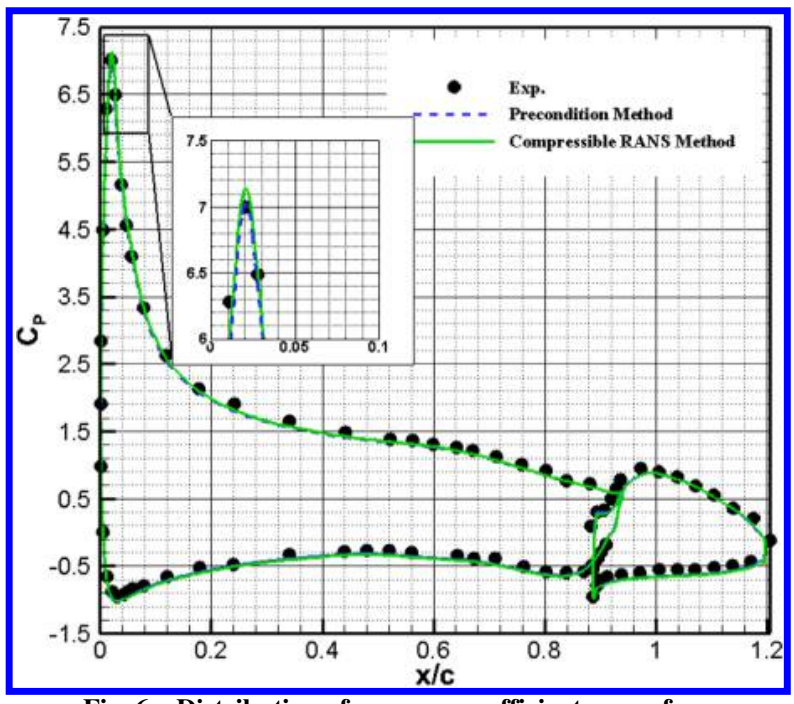

Fig. 6 Distribution of pressure coefficient on surface.

The main element is NLR 7301 supercritical airfoil. A flap with 20 deg deflection angle is used, and the gap between the airfoil and the flap is $2.6 \%$ chord length of the main element. The experiment is carried out in the NLR $3 \times 2 \mathrm{~m}$ low-speed wind tunnel, with the inflow Mach number of 0.185 . The Reynolds number based on the chord length is $2.51 \times 10^{6}$, and the AOA is $6 \mathrm{deg}$.

Two simulations are performed with the traditional compressible RANS method and the preconditioned method, respectively. The preconditioned characteristic BCs for far-field boundaries are adopted, and the preconditioner parameter is taken as $M_{r}=0.4$.

The convergence histories of the lift coefficient are plotted in Fig. $\underline{5}$. The lift coefficient convergences when the fluctuation between two adjacent iterations is less than $1 \times 10^{-5}$. It shows that the result of the preconditioned method converges after about 4000 iterations, whereas the lift coefficient given by the compressible RANS method does not converge until 7000 iterations. The lift coefficient predicted by the traditional compressible RANS method is 2.4656 , which is overpredicted compared with the experimental value of 2.41 . The result provided by the preconditioned method is 2.3945 and is in better agreement with the experimental data. Figure 6 depicts the comparison of the pressure coefficient distribution on the wall surface. Pressures predicted by both methods are in good agreement with the experimental data.

The velocity profiles are plotted at four typical locations in the boundary layer, the positions of which are sketched in Fig. 7. Points 1
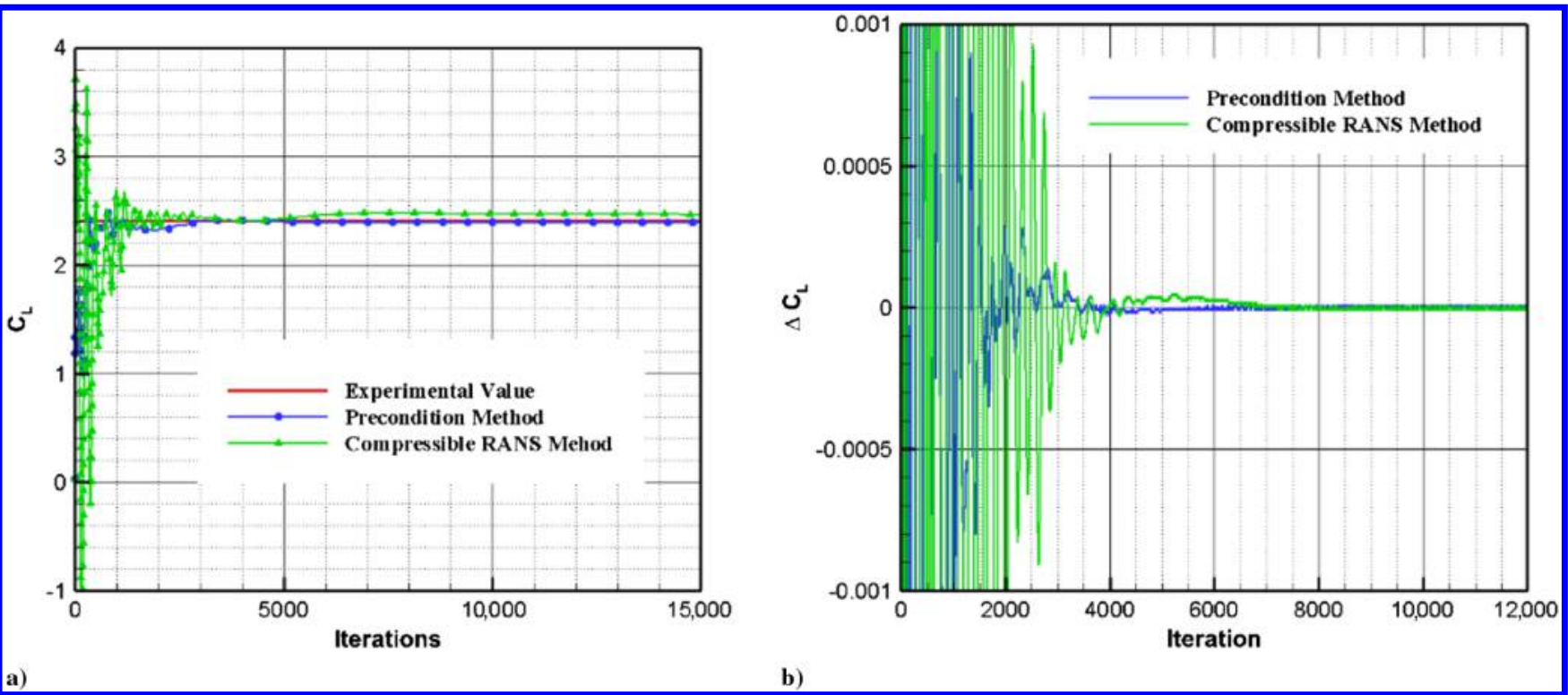

Fig. 5 Lift coefficient convergence history: a) time history, and b) fluctuation history. 


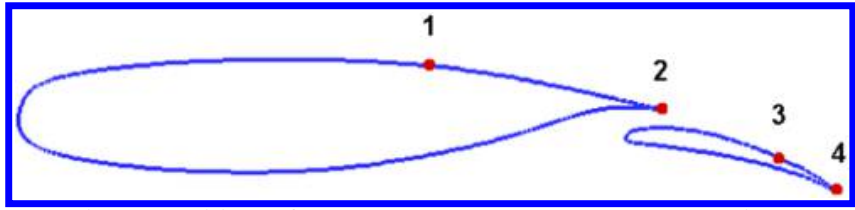

Fig. 7 Positions of the boundary-layer measurement stations.

and 2 are located on the upper surface of the main element, monitoring the velocity at $60 \%$ chord length of the airfoil and at the trailing edge, respectively. Points 3 and 4 are located on the upper surface of the flap, monitoring the velocity at the $60 \%$ chord length of the flap and at the trailing edge, respectively. The predicted results are compared with the measurement data in Fig. 8 .

The thickness of the boundary layer grows along the flow direction on the main element (at positions 1 and 2), and an obvious velocity defect in the wake of the main element appears at positions 3 and 4. Results of both methods are similar and agree well with the experimental data.

The comparison of the pressure distribution on the wall and velocity profiles in the boundary layers show that both of the solvers provide preferable results compared with the experimental data. Compared with the traditional compressible RANS method, the preconditioned solver with the preconditioned characteristic BCs significantly speed up the convergence rate of the simulation.

\section{High-Lift Trap-Wing Configuration}

Low-Mach-number flow over a 3-D high-lift trap-wing configuration is analyzed with the preconditioned method to validate its ability to solve complex problems. The complex geometry shape of the configuration makes the flow physics challenging for CFD solvers. Those challenges include massive flow separations on the flap, boundary-layer transitions, wake interactions, and the wing-tip vertical flow due to the changes of working conditions. The traditional compressible RANS solver performs terrible convergence behavior for such problems; thus, a robust and accurate CFD solver is essential for the study of the configuration.

Two test cases are analyzed in this section. In case 1 , flow around the configuration at the AOA of $\alpha=13 \mathrm{deg}$ is simulated with two meshes (the coarse mesh has 5.96 million cells, and the medium mesh has 20.1 million cells). Then, deflection predictions at four angles of attack ranging from $\alpha=-3.834 \mathrm{deg}$ to $\alpha=-28 \mathrm{deg}$ are computed on the coarse mesh. For both of the test cases, the inflow Mach number is $M_{\infty}=0.2$, and the Reynolds number based on the mean aerodynamic chord length is $R e=4.3 \times 10^{6}$. The preconditioner

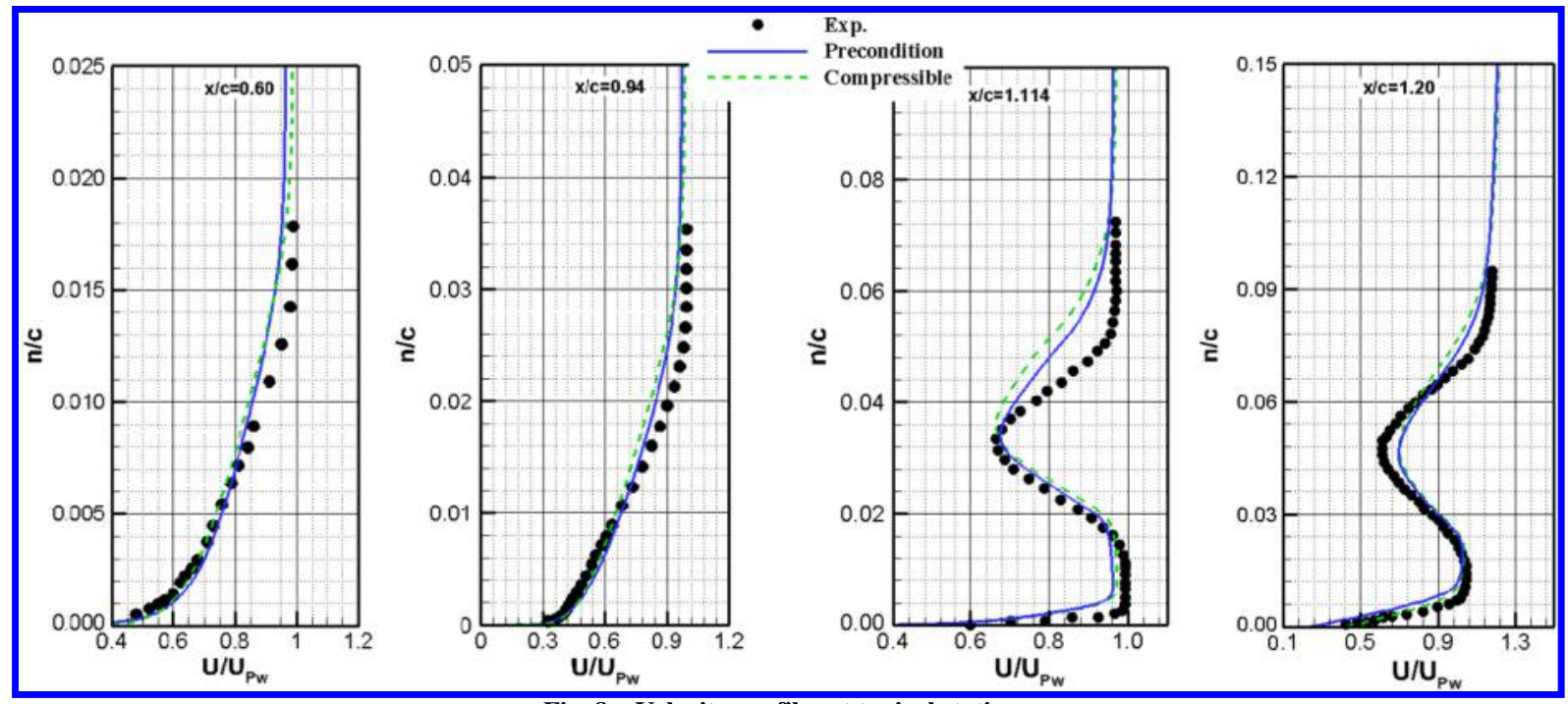

Fig. 8 Velocity profiles at typical stations.

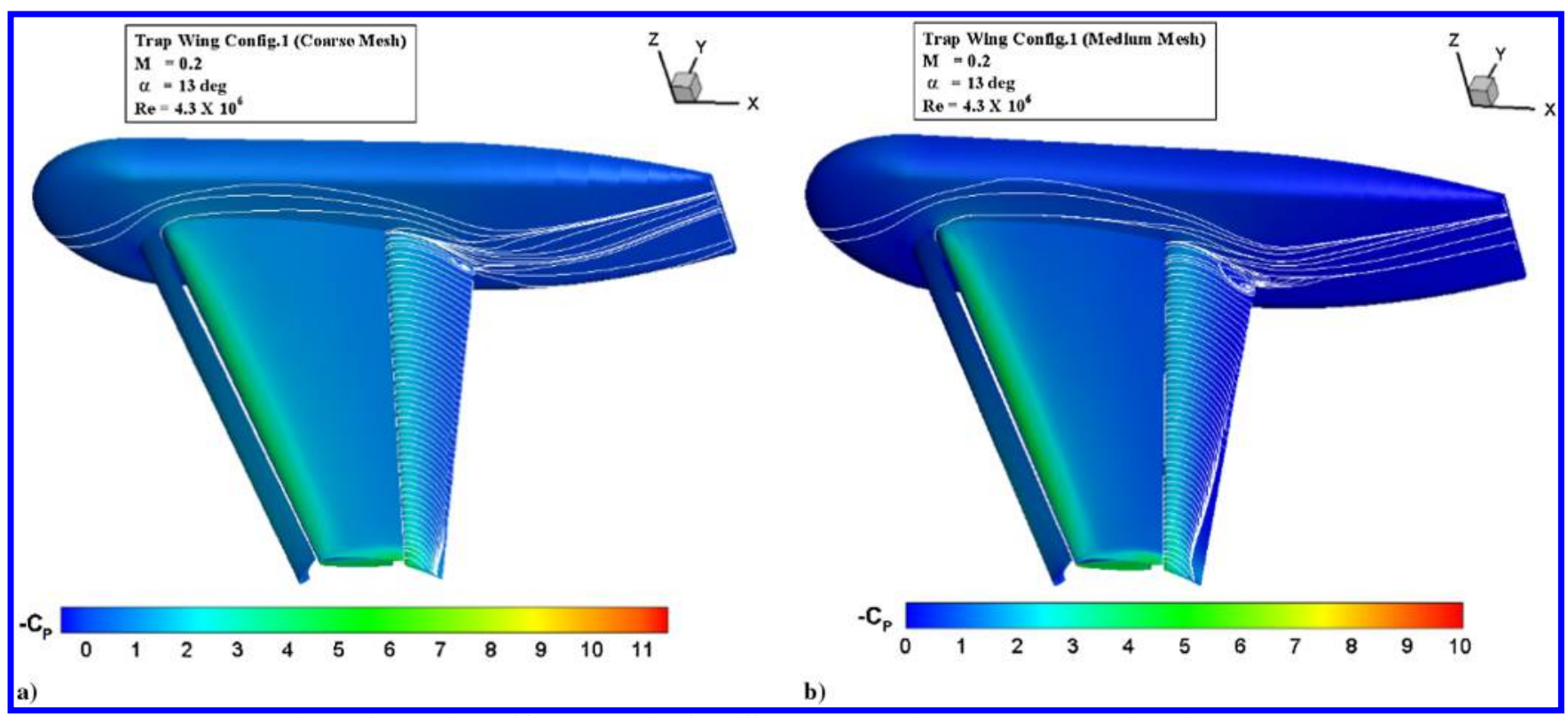

Fig. 9 Comparison of flap separation for different meshes: a) coarse mesh, and b) medium mesh. 

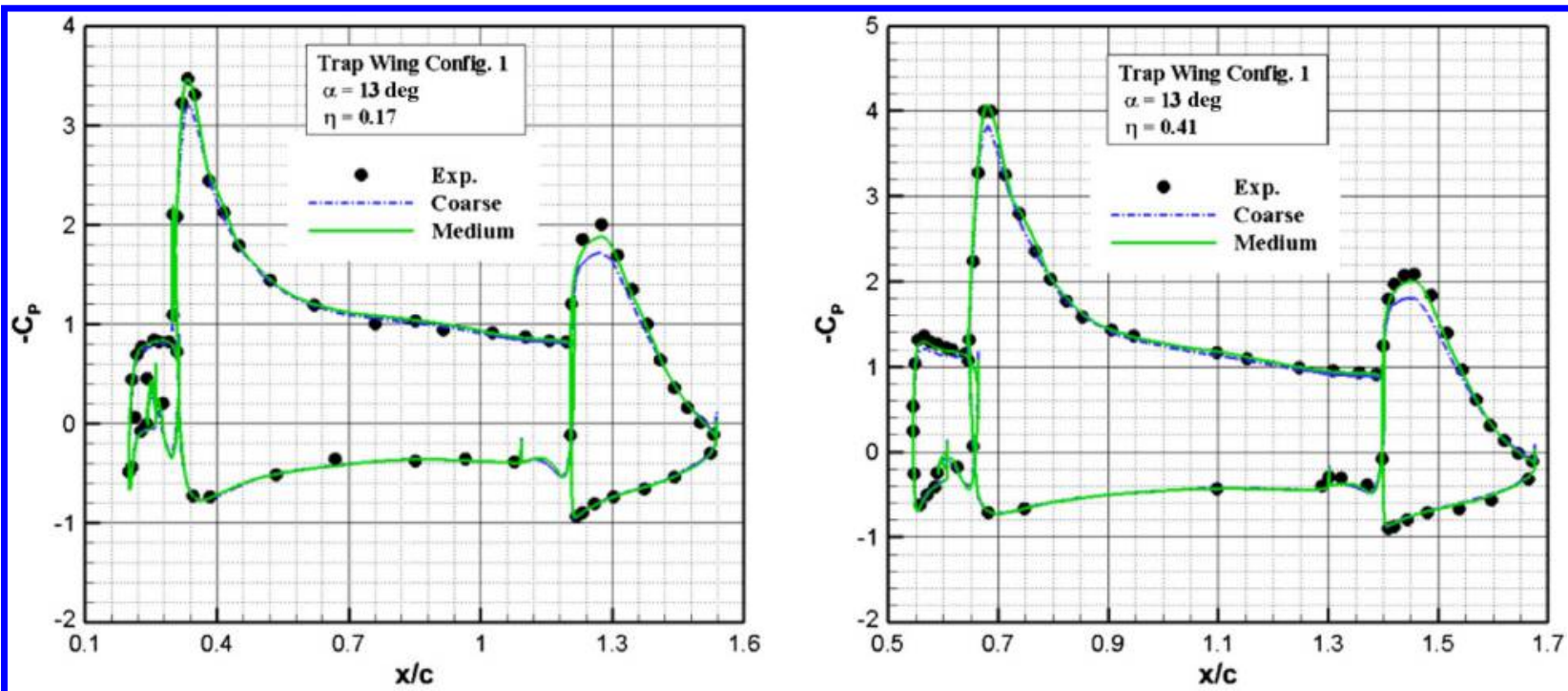

a)

b)
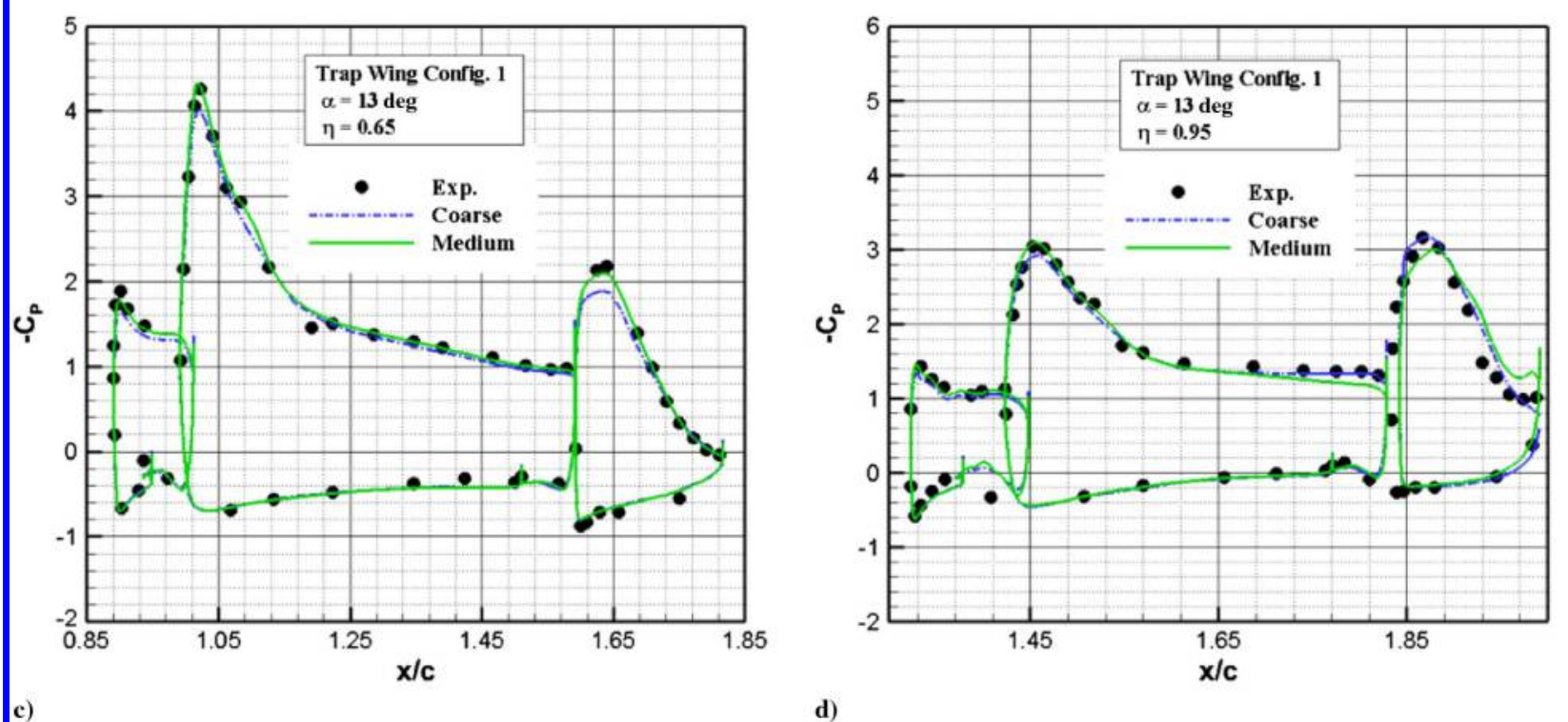

Fig. 10 Surface pressure distribution at different stations: a) 17\% spanwise, b) $41 \%$ spanwise, c) 65\% spanwise, and d) $95 \%$ spanwise.
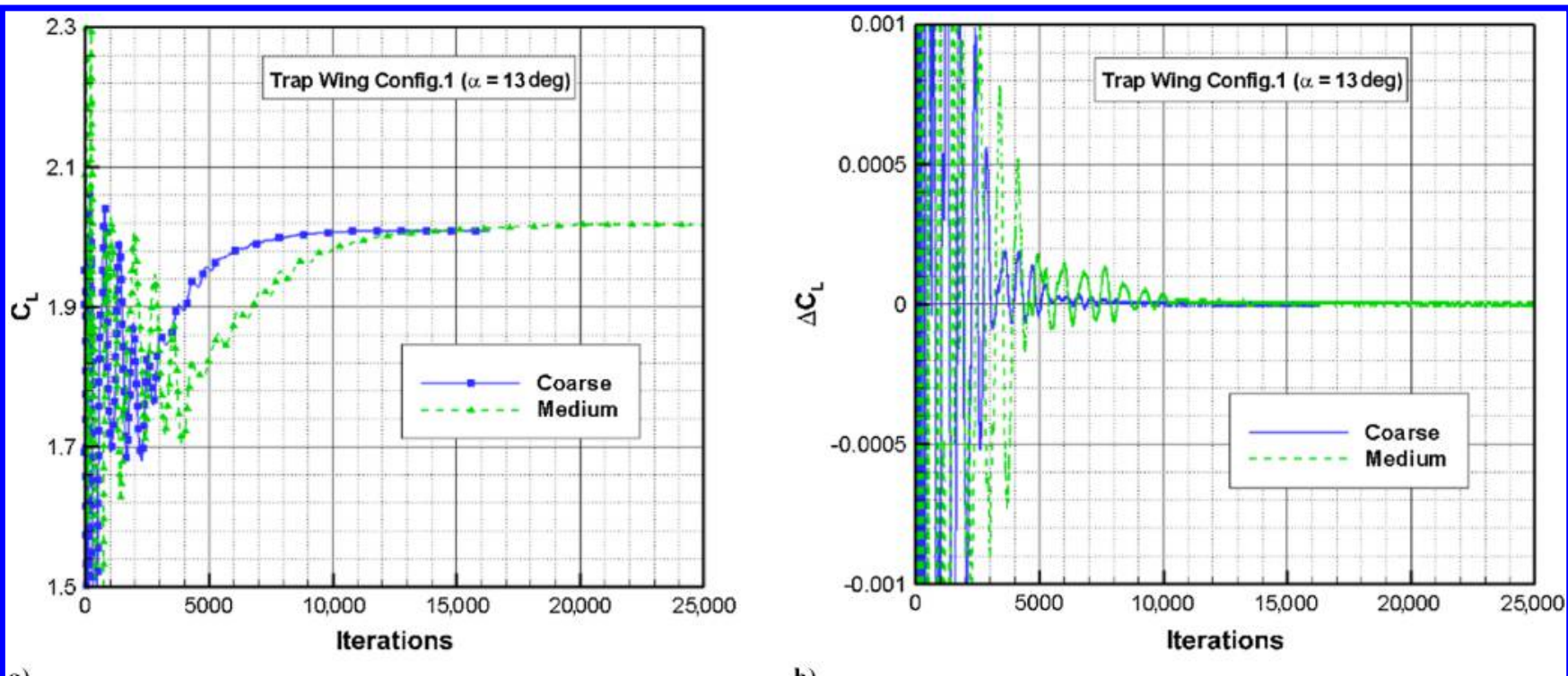


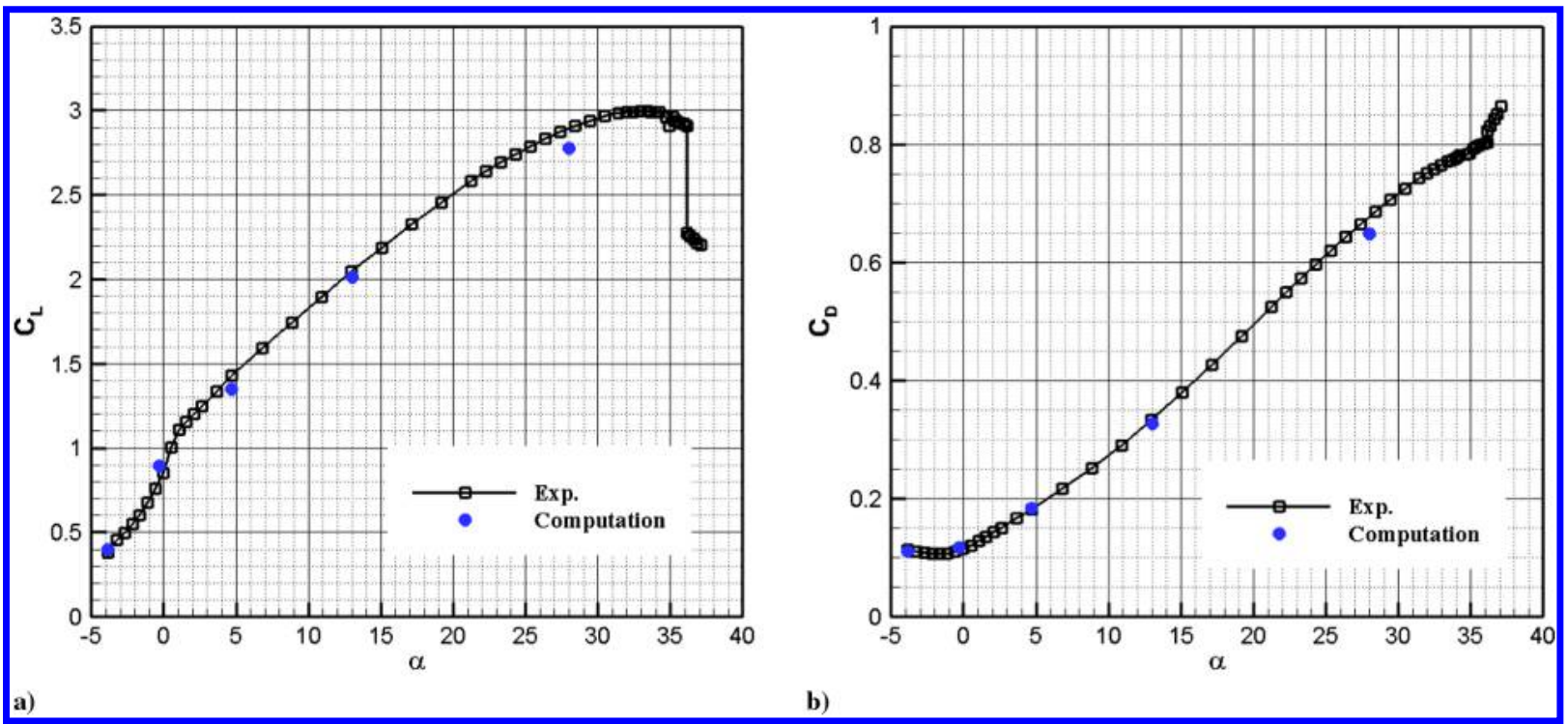

Fig. 12 Lift and drag coefficient vs angle of attack.

parameter is taken as $M_{r}=0.4$, and the preconditioned characteristic $\mathrm{BCs}$ are implemented on the far-field boundaries.

Figure 9 shows the comparison of surface streamlines on the flap at AOA of $\bar{\alpha}=13 \mathrm{deg}$. The separation region on the flap trends to be larger when the mesh is refined, and a similar phenomenon exists for the separation region at the flap root. Figure 10 plots the pressure distribution over high-lift surfaces on four different cross sections. Results provided by both the coarse and the medium mesh agree well with the experimental data on the lower surface. On the upper surface, the medium mesh provides better prediction compared with the measurement data.

The lift coefficient convergence histories at the AOA of $13 \mathrm{deg}$ for both the meshes are plotted in Fig. 11. It shows that the result of the coarse mesh converges after about 10,000 iterations, and the lift coefficient predicted by the medium mesh converges at about 15,000 iterations. The lift given by the coarse mesh is 2.0079 , and the medium mesh is 2.0137 . The lifts of both the meshes agree well with the experiment data, which is given as 2.0468 .

The aerodynamic forces with respect to the AOA are plotted in Fig. 12. The predicted lift coefficients agree well with the measurement with the AOAs less than $13 \mathrm{deg}$. The result at $\alpha=28 \mathrm{deg}$ is much underpredicted, with a deviation of $3.4 \%$. A similar trend can be found for the drag coefficients; the drag coefficient at $\alpha=28 \mathrm{deg}$ is underpredicted, whereas results at other angles of attack agree well with the measurement data.

\section{Conclusions}

A cell-centered finite-volume, three-dimensional preconditioned flow solver with the Weiss-Smith preconditioner is developed for lowMach-number flow simulations. A preconditioned HLLEW scheme with modification of its dissipation term is rederived to reduce the overlarge numerical dissipation. Preconditioned characteristic BCs based on the Weiss-Smith preconditioner are derived at the far-field boundaries, and the preconditioned LU-SGS implicit time-marching method is adopted to improve the convergence rate of the solution. Flow over the RAE 2822 airfoil is simulated to assess the effect of the preconditioned characteristic $\mathrm{BCs}$, and two high-lift configurations in 2-D and 3-D are analyzed with the solver.

The effect of the preconditioned characteristic BCs is assessed by the comparisons with the simplified $\mathrm{BCs}$ and the standard Riemann BCs. It is found that the boundary conditions on the far-field boundaries have an important influence on the performance of the flow solver. The study indicates that the preconditioned characteristic $\mathrm{BCs}$ eliminate the influence of the reflection spurious waves on the far-field boundaries and significantly increase the efficiency of the solver compared with the simplified BCs. Compared with the standard Riemann BCs, the preconditioned characteristic BCs gives better prediction results, though its efficiency improvement is inferior to that of the standard Riemann BCs.

Compared with the traditional compressible RANS solver, the preconditioned solver with the preconditioned characteristic BCs significantly speed up the convergence rate of the simulation for lowspeed flows around high-lift configurations. The comparison of the pressure distribution on the wall and the velocity profiles in the boundary layers shows that both of the solvers provide preferable results compared with experimental data. For the high-lift configuration with complex geometry, the preconditioned flow solver with the preconditioned characteristic BCs can provide accurate prediction with efficiency and achieve better robustness, whereas the compressible RANS solver led to diverged solutions with the same computational conditions.

\section{Acknowledgments}

This work was supported by the National High-Tech Research and Development Program of China 2014AA110501.

\section{References}

-11] Chorin, A. J., "A Numerical Method for Solving Incompressible Viscous Flow Problems," Journal of Computational Physics, Vol. 52, No. 6, Aug. 1997, pp. 118-125. doi:10.1006/jcph.1997.5716

-[2] Turkel, E., "Preconditioned Methods for Solving the Incompressible and Low Speed Compressible Equations," Journal of Computational Physics, Vol. 72, No. 6, Oct. 1987, pp. 277-298. doi:10.1016/0021-9991(87)90084-2

[3] Van Leer, B., Lee, W. T., and Roe, P. L., "Characteristic Time-Stepping or Local Preconditioning of the Euler Equations," 10th Computational Fluid Dynamics Conference, AIAA Paper 1991-1552, 1991.

[4] Weiss, J. M., and Smith, W. A., "Preconditioning Applied to Variable and Constant Density Flows," AIAA Journal, Vol. 33, No. 11, Nov. 1995, pp. 2050-2057. doi: $10.2514 / 3.12946$

[5] Venkatakrishnan, V., "Preconditioned Conjugate Gradient Methods for the Compressible Navier-Stokes Equations," AIAA Journal, Vol. 29, No. 7, July 1991, pp. 1092-1100. doi: $10.2514 / 3.10708$

[6] Choi, Y., and Merkle, C. L., "The Application of Preconditioning in Viscous Flows," Journal of Computational Physics, Vol. 105, No. 6, April 1993, pp. 207-223. doi:10.1006/jcph.1993.1069

- [7] Djeddi, R., and Ekici, K., "A Turbulent Low-Speed Preconditioner for Unsteady Flows About Wind Turbine Airfoils," 22nd AIAA Computational Fluid Dynamics Conference, AIAA Paper 2015-3202, June 2015.

- [8] Edwards, J. R., and Liou, M., "Low-Diffusion Flux-Splitting Methods for Flows at All Speeds," AIAA Journal, Vol. 36, No. 9, Sept. 1998, 
pp. $1610-1617$.

doi: $10.2514 / 2.587$

[9] Mark, P., Venkateswaran, S., and Shishir, P., "Unsteady Low Mach Preconditioning with Application to Rotorcraft Flows," 18th AIAA Computational Fluid Dynamics Conference, AIAA Paper 2007-4473, June 2007.

[10] Park, S. H., Lee, J. E., and Kwon, J. H., "Preconditioned HLLE Method for Flows at All Mach Numbers," AIAA Journal, Vol. 44, No. 11, Nov. 2006, pp. 2645-2653.

doi:10.2514/1.12176

[11] Turkel, E., Radespiel, R., and Kroll, N., "Assessment of Preconditioning Methods for Multidimensional Aerodynamics," Computers and Fluids, Vol. 26, No. 6, 1997, pp. 613-634. doi:10.1016/S0045-7930(97)00013-3

[12] Hejranfar, K., and Kamali-Moghadam, R., "Preconditioned Characteristic Boundary Conditions for Solution of the Preconditioned Euler Equations at Low Mach Number Flows," Journal of Computational Physics, Vol. 231, No. 12, June 2012, pp. 4384-4402. doi:10.1016/j.jcp.2012.01.040

[13] Turkel, E., "Preconditioning Techniques in Computational Fluid Dynamics," Annual Review of Fluid Mechanics, Vol. 31, Jan. 1999, pp. $385-416$. doi:10.1146/annurev.fluid.31.1.385

[14] Turkel, E., and Vatsa, V. N., "Local Preconditioners for Steady State and Dual Time-Stepping," ESAIM: Mathematical Modeling and Numerical Analysis, Vol. 39, No. 3, 2005, pp. 515-536.

[15] Turkel, E., "Preconditioning Methods for Low-Speed Flows," 14th Applied Aerodynamics Conference, AIAA Paper 1996-2460, 1996.
[16] Liu, Z., and Ramakrishnan, S. V., "Preconditioned Upwind Schemes for Simulation of Low Speed Flows on Hybrid Grids," 42nd AIAA Aerospace Sciences Meeting and Exhibit, AIAA Paper 2004-1268, Jan. 2004.

[17] Obayashi, S., and Wada, Y., "Practical Formulation of a Positively Conservative Scheme," AIAA Journal, Vol. 32, No. 5, May 1994, pp. 1093-1095. doi: $10.2514 / 3.12104$

[18] Yang, G., Obayashi, S., and Nakamichi, J., "Aileron Buzz Simulation Using an Implicit Multiblock Aeroelastic Solver," Journal of Aircraft, Vol. 40, No. 3, May 2003, pp. 580-589. doi: $10.2514 / 2.3134$

[19] Alexandre, A., Joao, L. A., and Ricardo, D. S., "Numerical Simulations of Turbulent Flow over a High-Lift Configuration," 29th AIAA Applied Aerodynamics Conference, AIAA Paper 2011-3006, June 2011.

[20] Stuart, R., Karlin, R., and Steven, N., "CFD Validation of High-Lift Flows with Significant Wind-Tunnel Effects," 18th Applied Aerodynamics Conference, AIAA Paper 2000-4218, 2000.

[21] Mark, C., and Shahyar, P, "Unstructured Navier-Stokes High-Lift Computations on a Trapezoidal Wing," 23rd AIAA Applied Aerodynamics Conference, AIAA Paper 2005-5084, June 2005.

[22] Rumsey, C. L., Slotnick, J. P., Long, M., Stuever, R. A., and Wayman, T. R., "Summary of the First AIAA CFD High-Lift Prediction Workshop," Journal of Aircraft, Vol. 48, No. 6, Nov. 2011, pp. 20682079.

doi:10.2514/1.C031447 